\title{
Role of reactive oxygen species in the anticancer activity of botanicals: Comparing sensitivity profiles
}

\author{
ZOYA COHEN $^{1}$, YAIR MAIMON ${ }^{1}$, NOAH SAMUELS ${ }^{1}$ and RAANAN BERGER ${ }^{2}$ \\ ${ }^{1}$ Tal Center for Integrative Oncology, Institute of Oncology; ${ }^{2}$ Institute of Oncology, \\ Sheba Medical Center, 52621 Ramat Gan, Israel
}

Received February 16, 2016; Accepted June 27, 2016

DOI: $10.3892 / \mathrm{ol} .2017 .5747$

\begin{abstract}
Numerous botanicals have been shown to exhibit in vitro and in vivo anticancer activity, some of which is the result of the induction of reactive oxygen species (ROS) in cancer cells with a high ROS content. The present study compared sensitivities to a series of botanicals among cancer cell lines, using an XTT viability test, in order to create a specific cancer-herb profile. Of the 27 botanicals screened, 10 exhibited a cytotoxic effect, 7 of which were ROS-mediated. The sensitivity profiles of the ROS-inducing botanicals in 10 cancer cell lines were similar, unlike 3 cytotoxic ROS-independent botanicals that displayed divergent botanical-specific profiles. The correlation between sensitivity profiles of ROS-inducing botanicals suggests a common mechanism of action, in contrast to the varied mechanism of ROS-independent botanicals. This implies that the investigation of the anticancer activity of botanicals should start with the examination of ROS-mediated activity. Further investigation of ROS sensitivity among various tumor types is required in order to guide research into developing evidence-based guidelines in the use of botanicals for cancer treatment.
\end{abstract}

\section{Introduction}

For thousands of years mankind has been using botanicals in order to alleviate suffering and heal disease, including cancer. A number of today's anticancer agents, including paclitaxel (Taxol), vinca alkaloids, etoposide and camptothecin, are themselves derived from plants (1), and much research is taking place on the use of these products for the treatment of cancer. The findings of this research are providing a growing amount of evidence indicating that certain botanicals are safe and

Correspondence to: Dr Yair Maimon, Tal Center for Integrative Oncology, Institute of Oncology, Sheba Medical Center, 2 Derech Sheba Road, Tel Hashomer, 52621 Ramat Gan, Israel

E-mail: yair@tcm.org.il

Key words: reactive oxygen species, botanical, sensitivity profile, cytotoxic, cancer effective in promoting immune-modulatory effects, reducing chemotherapy-induced toxicities (e.g., hematological and clinical), and most importantly, exhibiting direct anticancer properties $(2,3)$. More recent research has begun to investigate the mechanisms of action of selected botanicals, isolating the active components of traditionally used botanicals and establishing specific anticancer activity profiles. The ultimate goal of this research is to provide guidelines for the integration of botanical medicine within the accepted standard-of-care treatment of cancer, and to further aid in promoting the development of anticancer drugs.

Reactive oxygen species (ROS) are chemically active oxygen-containing molecules, with either unpaired electron(s) (free radicals) or paired electrons, which can be converted into a free radical form (4). ROS are produced naturally during mitochondrial respiration and other intracellular processes. Despite the common belief that free radicals have a deleterious effect on health, ROS play an important role as transducers in a number of signaling pathways that are vital to cell differentiation, proliferation and survival. At the same time, elevated ROS levels can often be found in cancer cells; this is due to either a compromised ROS-scavenging capacity or increased ROS production (4). Increased ROS activity can drive cancer cells to abnormal proliferation, while at the same time bringing them closer to a cell-death threshold, at which stage ROS can induce irreversible cell damage (4). In recent years the role of ROS has become an important focus of cancer research, with a potential for the development of novel anticancer therapies.

Numerous medicinal plants with known anticancer activity have been shown to induce cancer cell death through ROS induction, most likely due to their pro-oxidant flavonoid components (5). An example of this is quercetin, a flavonoid found in a number of medicinal plant products, as well as certain fruits, vegetables and aromatic plants (5). The selective anticancer effect of ROS-inducing compounds reflects the presence of higher baseline levels of ROS in cancer cells compared with non-cancer cells, bringing them closer to the cell-death threshold and increasing their sensitivity to ROS formation $(4,5)$. Thus, the sensitivity of cells to ROS induction appears to be dependent primarily on the ROS levels of the individual cells being targeted. As such, by exposing a panel of cancer cell lines to various ROS-inducing botanicals, researchers could theoretically be able to derive a sensitivity 
profile that reflects the sensitivity of tested cell lines to ROS, which is similar to all ROS-inducing botanicals. At the same time, analogous testing of ROS-independent botanicals could provide a unique sensitivity profile for each botanical.

In order to test the aforementioned hypothesis, the cytotoxic effects of 27 botanicals on a panel of 10 cancer cell lines were investigated. The products studied were chosen in accordance with the principles of traditional Chinese medicine herbology, as well as findings from our previous studies (6). The botanicals that exhibited clear anticancer activity were then tested against the same panel of cells, this time using the ROS scavenger pyruvate. In this way, the study was able to distinguish between ROS-dependent and -independent cytotoxicity. The sensitivity profiles for the two groups of botanicals (ROS-dependent and -independent) were then compared.

\section{Materials and methods}

Cancer cell line cultures. The following cancer cell line cultures were used in the study: A549 lung carcinoma, MCF7 breast adenocarcinoma, MDA-MB-231 breast adenocarcinoma, PC-3 prostate adenocarcinoma, DU-145 prostate carcinoma, T24 bladder transitional cell carcinoma and PANC-1 pancreas epithelioid carcinoma cell lines, which were obtained from American Type Culture Collection (Manassas, VA, USA); SK-N-BE(2) neuroblastoma cell line, which was provided by Dr. Rinat Abramovitch (Hadassah Hebrew University Medical Center, Jerusalem, Israel); and human $526 \mathrm{mel}$ and $624 \mathrm{mel}$ melanoma cell lines, which were provided by Dr Gal Markel (Ella Institute of Melanoma, Sheba Medical Center, Ramat-Gan, Israel). All cell lines were propagated in RPMI 1640 supplemented with $10 \%$ fetal bovine serum, $2 \mathrm{mM}$ L-glutamine and $100 \mu \mathrm{g} / \mathrm{ml}$ penicillin/streptomycin (Biological Industries, Beit Ha-Emek, Israel), and incubated at $37^{\circ} \mathrm{C}$, in a $5 \% \mathrm{CO}_{2}$ atmosphere.

Botanical extracts. Standardized dried herbal extracts were purchased from Zen Herbs (Tel Aviv, Israel), dissolved in phosphate-buffered saline (PBS) at a concentration of $100 \mathrm{mg} / \mathrm{ml}$, and incubated at $60^{\circ} \mathrm{C}$ for $30 \mathrm{~min}$ with occasional application of a vortex. The solution was centrifuged at $2,700 \mathrm{x} \mathrm{g}$ for $5 \mathrm{~min}$, and the supernatant was filtered through a $0.45-\mu \mathrm{M}$ Millex polyvinylidene difluoride filter (Merck Millipore, Carrigtwohill, Ireland). Solubility was estimated by cryophilization and weighing of the pellet, and was estimated to be $\sim 50 \%$. For convenience, the final stock concentration was designated at $100 \mathrm{mg} / \mathrm{ml}$ (w/v concentration of dried powder in PBS) enabling the comparison of the individual herbal components. The acronyms of the botanicals used throughout the present study are listed in Table I.

Cytotoxicity screen and XTT viability assay. The cells were plated at a density of 3,000 cells/well in triplicate over 96-well plates in RPMI-1640 supplemented with $10 \%$ fetal bovine serum, $2 \mathrm{mM}$ L-glutamine and $100 \mu \mathrm{g} / \mathrm{ml}$ penicillin/streptomycin (Biological Industries, Beit Ha-Emek, Israel) and allowed to attach and grow overnight at $37^{\circ} \mathrm{C}$, in a $5 \% \mathrm{CO}_{2}$ atmosphere. The medium was replaced with a fresh treatment-containing medium, and the cells were propagated
Table I. Botanicals used in the study $(n=27)$.

\begin{tabular}{|c|c|}
\hline Acronym & Latin binomial \\
\hline AAS & Anemarrhena asphodeloides \\
\hline AMA & Atractylodes macrocephala \\
\hline $\mathrm{AME}$ & Astragalus membranaceus \\
\hline CRE & Citrus reticulata \\
\hline GLI & Glehnia littoralis \\
\hline GLU & Ganoderma lucidum \\
\hline $\mathrm{HCO}$ & Houttuynia cordata \\
\hline LPA & Loranthus parasiticus \\
\hline ITI & Isatis tinctoria \\
\hline $\mathrm{LCH}$ & Lycium chinense \\
\hline ODI & Oldenlandia diffusa \\
\hline OSA & Oryza sativa germinatus \\
\hline OJA & Ophiopogon japonicus \\
\hline $\mathrm{PCO}$ & Poria cocos \\
\hline PGI & Panax ginseng \\
\hline PLR & Paeonia lactiflora red \\
\hline PLW & Paeonia lactiflora white \\
\hline PQU & Radix Panacis quinquefolii \\
\hline PCP & Poria cocos paradicis \\
\hline PVU & Prunella vulgaris \\
\hline SBA & Scutellaria barbata \\
\hline $\mathrm{SCB}$ & Scutellaria baicalensis \\
\hline SFL & Radix Sophora flavescentis \\
\hline SGL & Radix Smilax glabra \\
\hline SSU & Spatholobus suberectus \\
\hline STO & Sophora tonkinensis \\
\hline VHI & Vaccaria hispanica \\
\hline
\end{tabular}

for an additional $48 \mathrm{~h}$ in the same conditions. In experiments with pyruvate and N-acetyl-cysteine (NAC), $1 \mathrm{mM}$ pyruvate or $10 \mathrm{mM}$ NAC were added to the medium together with botanical extracts, mixed and then added to the cells. An XTT viability test was performed by replacing the medium with fresh RPMI-1640 supplemented with $10 \%$ fetal bovine serum, $2 \mathrm{mM}$ L-glutamine and $100 \mu \mathrm{g} / \mathrm{ml}$ penicillin/streptomycin, to prevent interference of treatment color with XTT reagent signal, and adding XTT reagent (Biological Industries) for incubation for 2-3 h. The blank measurement was subtracted from each reading, and all values of treated cells were divided by control (PBS-treated cells) values in each experiment (control=1). The resulting signal was measured by an enzyme-linked immunosorbent assay reader. Each experiment was repeated at least three times.

Statistical analysis. The mean \pm standard deviation estimates were calculated from each experiment and performed in triplicate using Microsoft Excel 2007 (Microsoft, London, UK). The mean \pm standard deviation estimates in Fig. 1 (screening for anticancer activity) were calculated from at least three independent experiments. P-values were calculated using Student's 2-tailed T-test in Microsoft Excel 2007 program. Results were considered statistically significant if $\mathrm{P} \leq 0.05$. 

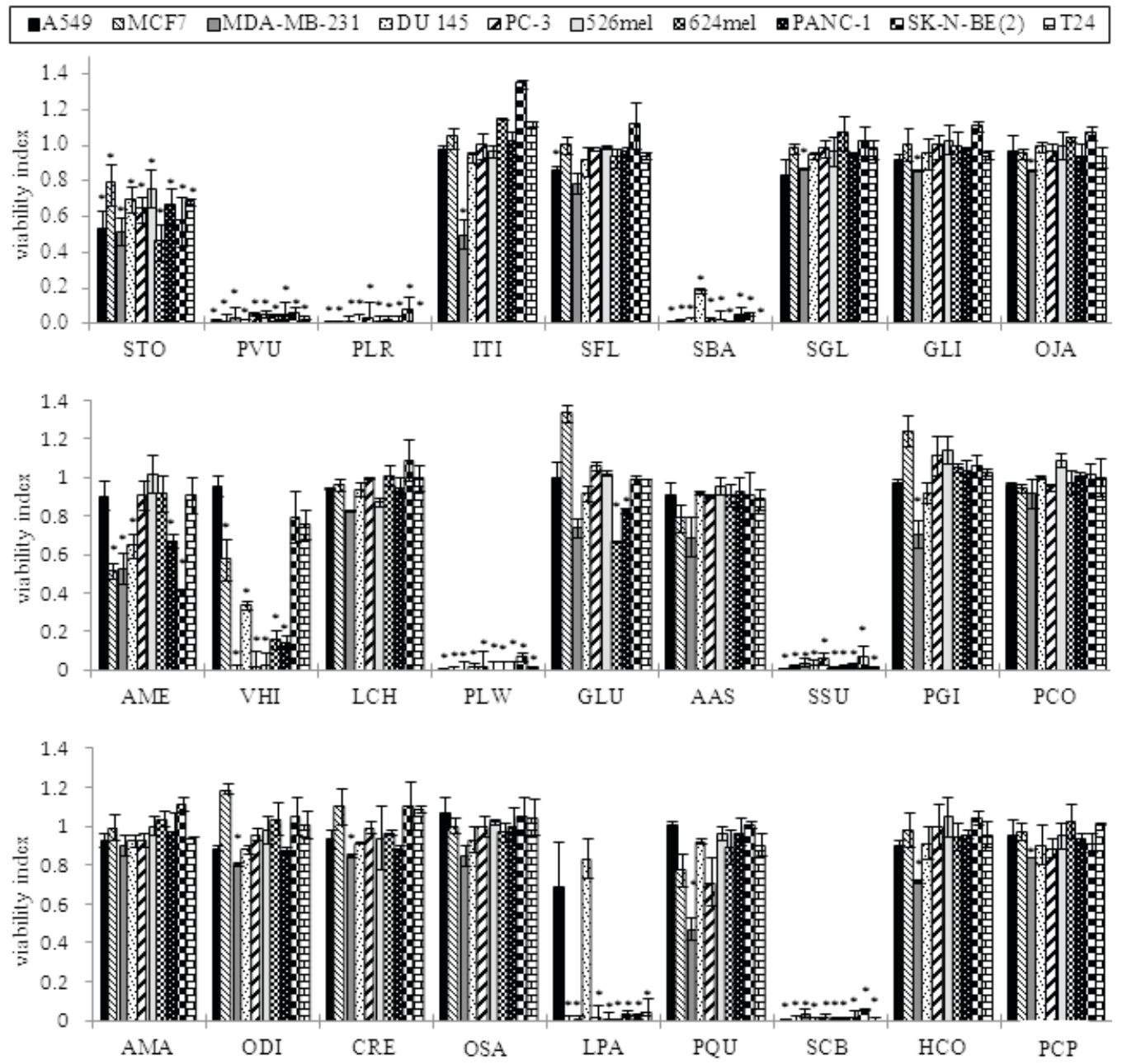

Figure 1. Cytotoxic screen. A total of 10 human cancer cell lines were plated at 3,000 cells/well in 96 -well plates and treated on the next day with $2 \mathrm{mg} / \mathrm{ml}$ of the freshly prepared indicated herbal extracts. After $48 \mathrm{~h}$ of treatment, the viability of the cells was assessed by an XTT viability assay. The graphs represent an average of at least three independent experiments. ${ }^{*} \mathrm{P}<0.05$. For the full name of the abbreviated botanicals see Table $\mathrm{I}$.

\section{Results}

Cytotoxic screen. The study first determined the concentration of the standardized botanical extracts for which clear cytotoxic activity was observed. For this purpose, MDA-MB-231 cells were treated with incremental concentrations of 14 herbal compounds, and $2 \mathrm{mg} / \mathrm{ml}$ was selected for further screening (data not shown). Next, 27 botanical compounds commonly used by practitioners of traditional Chinese medicine for the treatment of cancer were screened, examining their effect on a panel of the following 10 human cancer cell lines: A549 (lung), MCF7 and MDA-MB-231 (breast), DU-145 and PC-3 (prostate), 526mel and 624mel (melanoma), PANC-1 (pancreas), SK-N-BE (2) (neuroblastoma) and T24 (bladder). The following herbal compounds were found to exhibit significant anti-proliferative activity toward the cancer cells, and were selected for further study: Astragalus membranaceus (AME; A549, 0.0764; MCF7, 0.0026; MDA-MB-231, 0.0012; DU-145, 0.0013; PC-3, 0.12; Mel526, 0.768; Mel624,0.05; PANC-1, 0.05; SK-N-BE(2), 0.0098; T-24, 0.3801); Vaccaria hispanica (VHI; A549, 0.0252; MCF7, 0.003; MDA-MB-231, <0.001; DU-145, 0.0058; PC-3, 0.002; Mel526, 0.0012; Mel624,0.0012; PANC-1, 0.009; SK-N-BE(2), 0.17; T-24, 0.08); Paeonia lactiflora white (PLW; A549, 0.0028; MCF7, <0.001; MDA-MB-231, <0.001;
DU-145, <0.001; PC-3, <0.001; Mel526, <0.001; Mel624, $<0.001$; PANC-1, <0.001; SK-N-BE(2), <0.001; T-24, 0.001); Spatholobus suberectus (SSU; A549, <0.001; MCF7, $<0.001$; MDA-MB-231, <0.001; DU-145, <0.001; PC-3, $<0.001$; Mel526, <0.001; Mel624, <0.001; PANC-1, <0.001; SK-N-BE(2), <0.001; T-24, 0.001); Sophora tonkinensis (STO; A549, <0.001; MCF7, 0.03; MDA-MB-231, 0.001; DU-145, 0.003; PC-3, 0.002; Mel526, 0.02; Mel624, 0.001; PANC-1, 0.0026; SK-N-BE(2), 0.025; T-24, 0.03); Prunella vulgaris (PVU; A549, <0.001; MCF7, <0.001; MDA-MB-231, <0.001; DU-145, <0.001; PC-3, <0.001; Mel526, <0.001; Mel624, $<0.001$; PANC-1, <0.001; SK-N-BE(2), <0.001; T-24, <0.001); Paeonia lactiflora red (PLR; A549, 0.0026; MCF7, <0.001; MDA-MB-231, <0.001; DU-145, <0.001; PC-3, 0.003; Mel526, $<0.001$; Mel624, <0.001; PANC-1, <0.001; SK-N-BE(2), 0.001; T-24, 0.001); Scutellaria barbata (SBA; A549, 0.0016; MCF7, 0.023; MDA-MB-231, 0.0015; DU-145, 0.0049; PC-3, 0.0087; Mel526, <0.001; Me1624, <0.001; PANC-1, <0.001; SK-N-BE(2), <0.001; T-24, 0.001); Loranthus parasiticus (LTA; A549, 0.07; MCF7, 0.012; MDA-MB-231, <0.001; DU-145, 0.09; PC-3, 0.029; Mel526, 0.015; Mel624, <0.001; PANC-1, 0.0056; SK-N-BE(2), 0.033; T-24, 0.031); and Scutellaria baicalensis (SCB; A549, <0.001; MCF7, 0.0077; MDA-MB-231, <0.001; DU-145, 0.0047; PC-3, 0.009; Mel526, 

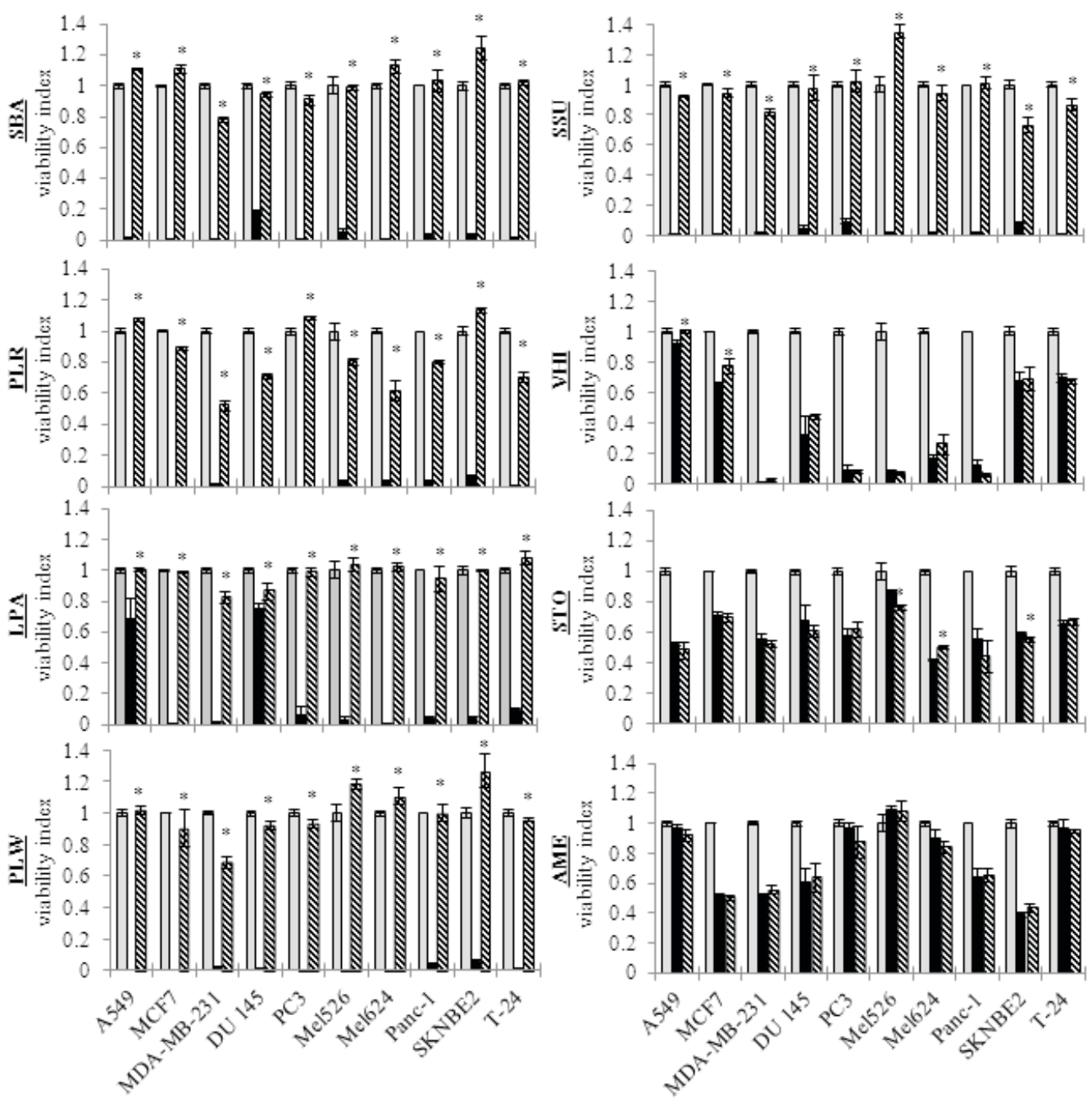

口control $\mathbf{a}$ herbal extract $\mathbf{\Delta}$ herbal extract + pyruvate

$\square$ control $\boldsymbol{w}$ herbal extract $\mathbf{\Delta h e r b a l}$ extract + pyruvate

Figure 2. Involvement of reactive oxygen species in the anticancer effect of toxic plants. Cells were plated at 3,000 cells/well in 96 -well plates and treated on the next day with $2 \mathrm{mg} / \mathrm{ml}$ of the freshly prepared indicated herbal extracts, either alone or in combination with $1 \mathrm{mM}$ of sodium pyruvate. Subsequent to $48 \mathrm{~h}$ of treatment, the viability of the cells was assessed by an XTT viability assay. For the full name of the abbreviated botanicals see Table I. * $<0.05$.

$<0.001 ; \mathrm{Mel624},<0.001 ; \mathrm{PANC}-1,<0.001 ; \mathrm{SK}-\mathrm{N}-\mathrm{BE}(2)$, $<0.001 ; \mathrm{T}-24,<0.001)$ (Fig. 1).

ROS-dependent anti-proliferative activity. In order to examine whether the anticancer toxicity of the selected botanicals was dependent on ROS induction, the panel of 10 cell lines was treated with pyruvate, a commonly used ROS scavenger (7). As shown in Fig. 2, the cytotoxic effects of 5 of the botanicals (SBA, LTA, PLW, PLR and SSU) were either completely or significantly reversed (50-100\% reversal) by the addition of pyruvate to the exposed culture, compared with botanical-only treatment. The P-values for the botanicals in the various cell lines were as follows: i) SBA: A549, <0.001; MCF7, <0.001; MDA-MB-231, <0.001; DU-145, <0.001; PC-3, $<0.001$; Mel526, <0.001; Mel624, <0.001; PANC-1, <0.001; SK-N-BE(2), <0.001; and T-24, <0.001; ii) LPA: A549, 0.014; MCF7, <0.001; MDA-MB-231, <0.001; DU-145, 0.036; PC-3, $<0.001$; Mel526, <0.001; Mel624, <0.001; PANC-1, <0.001; SK-N-BE(2), <0.001; and T-24,<0.001; iii) PLW: A549,<0.001; MCF7, <0.001; MDA-MB-231, <0.001; DU-145, <0.001; PC-3, $<0.001$; Mel526, <0.001; Mel624, <0.001; PANC-1, <0.001;
SK-N-BE(2), <0.001; and T-24, <0.001; iv) PLR: A549, <0.001; MCF7, <0.001; MDA-MB-231,<0.001; DU-145, <0.001; PC-3, $<0.001$; Mel526, <0.001; Mel624, <0.001; PANC-1, <0.001; SK-N-BE(2), <0.001; and T-24, <0.001; and v) SSU: A549, $<0.001$; MCF7, <0.001; MDA-MB-231, <0.001; DU-145, $<0.001$; PC-3, <0.001; Mel526, <0.001; Mel624, <0.001; PANC-1, <0.001; SK-N-BE(2), <0.001; and T-24, <0.001. This indicated that the anticancer effect of the botanicals was dependent on ROS induction in the targeted cells. The activity of 3 of the herbs (AME, STO and VHI) was not reversed by the presence of pyruvate (with few minor, $<15 \%$, exceptions), indicating that these botanicals were working through a non-ROS pathway. The P-values for A549, MCF7, MDA-MB231, DU-145, PC-3, Mel526, Mel624, PANC-1, SK-N-BE(2) and T-24, respectively, are: AME, 0.2, 0.36, 0.5, 0.49, 0.25, 0.67, 0.3, 0.74, 0.07 and 0.57; STO, 0.42, 0.31, 0.28, 0.39, 0.32, $<0.001,<0.001,0.18,0.009$ and 0.53 ; and VHI, 0.007, 0.04, 0.16, $0.32,0.55,0.36,0.07,0.18,0.87$ and 0.25 . Finally, 2 botanicals (PVU and SCB) displayed mixed results, with reversion of growth inhibition in the presence of pyruvate in certain cell lines (i.e., ROS-dependent) and with no or weak $(<10 \%)$ effect 
A
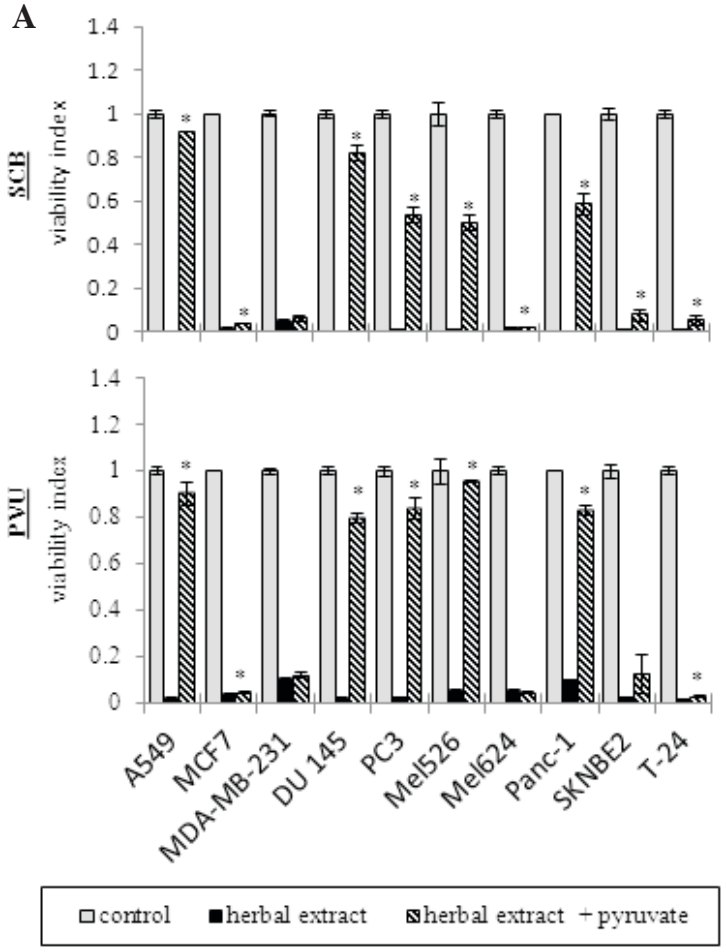
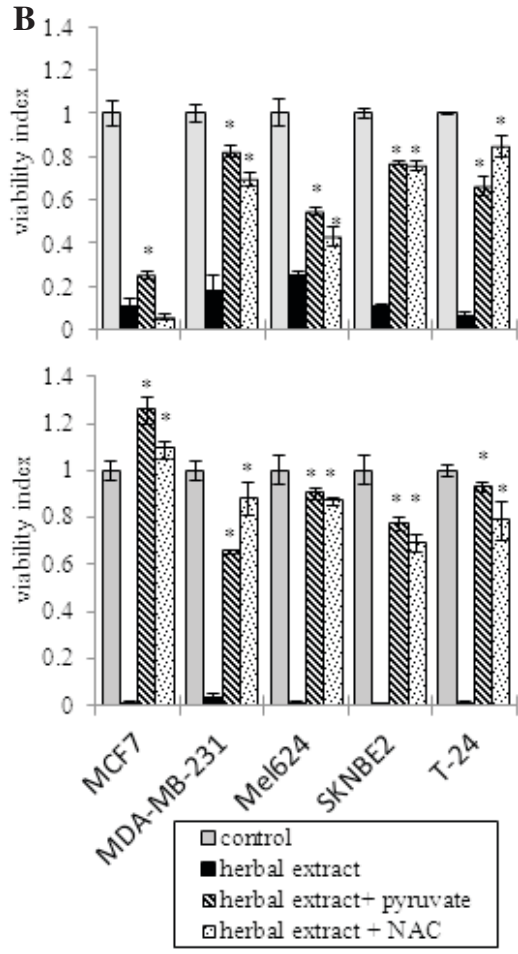

Figure 3. Involvement of reactive oxygen specied in the anticancer effect of Prunella vulgaris (PVU) and Scutellaria baicalensis (SCB). (A) Cells were plated as indicated and treated on the next day with $2 \mathrm{mg} / \mathrm{ml}$ of herbal extract, either alone or in combination with $1 \mathrm{mM}$ of sodium pyruvate. (B) Cells were plated as indicated and treated with $0.5 \mathrm{mg} / \mathrm{ml} \mathrm{PVU}$ or $0.25 \mathrm{mg} / \mathrm{ml} \mathrm{SCB}$ extract, either alone or in combination with $1 \mathrm{mM}$ of sodium pyruvate or $10 \mathrm{mM}$ of $\mathrm{N}$-acetyl-cysteine (NAC). Subsequent to $48 \mathrm{~h}$ of treatment, the viability of the cells was assessed by an XTT viability assay. For the full name of the abbreviated botanicals see Table I. ${ }^{*} \mathrm{P}<0.05$.

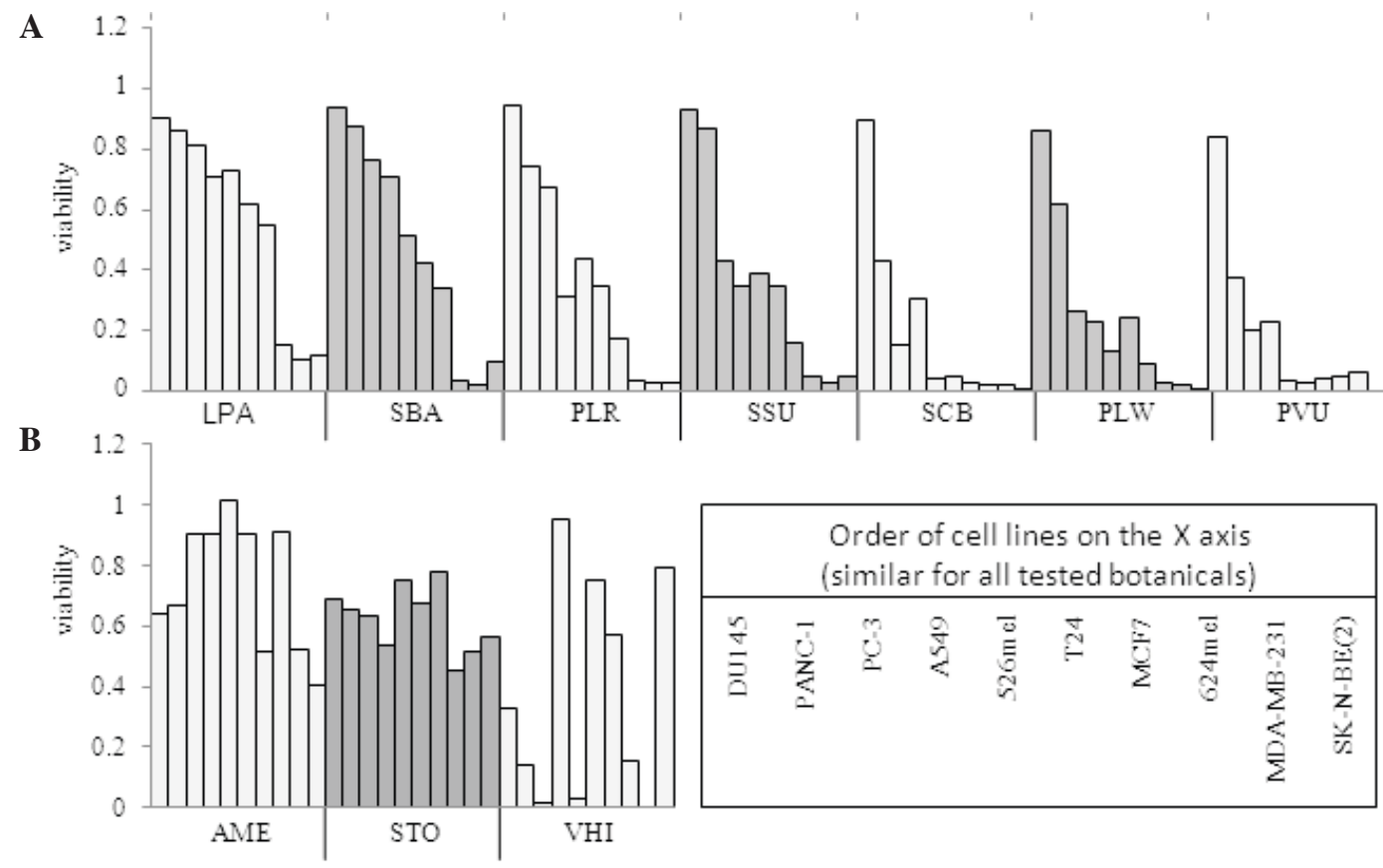

Figure 4. Sensitivity profiles. Cells were plated as indicated and treated on the next day with the following concentrations of herbal extracts: $2 \mathrm{mg} / \mathrm{ml}$ AME, VHI and STO; $1 \mathrm{mg} / \mathrm{ml} \mathrm{SBA}$ and LTA; $0.5 \mathrm{mg} / \mathrm{ml} \mathrm{PLW,} \mathrm{PLR,} \mathrm{SSU} \mathrm{and} \mathrm{PVU;} \mathrm{and} 0.25 \mathrm{mg} / \mathrm{ml} \mathrm{SCB}$. Subsequent to $48 \mathrm{~h}$ of treatment, the viability of the cells was assessed by an XTT viability assay. (A) Sensitivity profiles of 10 cancer cell lines to 7 reactive oxygen species (ROS)-inducing botanicals. (B) Sensitivity profiles of the same cells to 3 botanicals whose toxicity is ROS-independent. For the full name of the abbreviated botanicals see Table I.

of pyruvate in other cell lines (i.e., ROS-independent) (Fig. 3). The P-values for A549, MCF7, MDA-MB-231, DU-145, PC-3, Mel526, Mel624, PANC-1, SK-N-BE(2) and T-24, respectively, are: PVU, 10-8, 0.01, 0.051, <0.001, <0.001, <0.001, 0.41 , $<0.001,0.1$ and 0.009 ; and SCB, $<0.001,<0.001,0.27,<0.001$, $<0.001,<0.001,<0.001,<0.001,0.008$ and 0.024 . 
In order to test the hypothesis that the mixed effects in the last group of botanicals were the result of a greater ROS content, the experiment was repeated with lower concentrations of PVU and SCB, in the presence of either pyruvate or another ROS scavenger, NAC. This experiment was performed on those cells that were not protected by the addition of pyruvate in the earlier stage of the experiment. As shown in Fig. 3, the cytotoxic activity of PVU and SCB was either prevented or inhibited by the addition of the ROS scavengers, indicating that these botanicals were acting through ROS induction. The P-values for MCF7, MDA-MB-231, Mel624, SK-N-BE(2) and T-24, respectively, are: PVU+pyruvate, $<0.001,<0.001$, $<0.001,<0.001$ and $<0.001$; PVU+NAC, $<0.001,<0.001$, $<0.001,<0.001$ and $<0.001$; and SCB+pyruvate, $<0.001,0.002$, $<0.001,<0.001$ and $<0.001 ; \mathrm{SCB}+\mathrm{NAC},<0.001,0.06,0.004$, $<0.001$ and $<0.001$.

Sensitivity profile. In order to build a sensitivity profile, the concentration of each botanical was calibrated to enable the detection of the difference in the response between the cancer cell lines. For all of the 3 ROS-independent botanicals, a clear sensitivity profile was produced at $2 \mathrm{mg} / \mathrm{ml}$. By contrast, the concentration of each ROS-inducing botanical was reduced to $0.25-1 \mathrm{mg} / \mathrm{ml}$, in order to allow for the construction of a sensitivity profile. As shown in Fig. 4, all 7 ROS-inducing botanicals displayed a very similar sensitivity profile on the panel of the 10 cancer cell lines tested, with variation in potency. By contrast, the profiles of ROS-independent botanicals were all different from each other, as well as from the ROS-inducing botanicals.

\section{Discussion}

The present study found that ROS induction was a significant factor leading to cancer cell death for a number of the botanicals examined. While SBA, SSU and SCB have been shown to induce cell death through ROS induction (7-10), the anticancer effects of PLW and PLR and PVU $(11,12)$ have not been known to be ROS-dependent. While the anticancer effects o STO, AME and VHI have been reported elsewhere (13-16), their independence of ROS was demonstrated in the present study for the first time. The anticancer effects of LPA were also shown for the first time. It is notable that 7 out of the 10 botanicals that exhibited cytotoxic effects on the cancer cell lines were found to be ROS-dependent, indicating a prevalent mechanism of action for plant-derived products that are commonly used in the practice of traditional medicine. To the best of our knowledge, this is the first study to classify anti-cancer Chinese herbs according to their ROS activity.

In addition to classifying the botanicals according to their ROS-dependent and -independent cytotoxic activity, the present study showed that ROS-dependent botanicals had similar sensitivity profiles in a panel of 10 cancer cell lines. This was in contrast to the ROS-independent botanicals, which displayed divergent sensitivity profiles. These results confirmed our initial hypothesis regarding the sensitivity profiles of ROS-inducing botanicals, and suggested that a similar mechanism underlies their anticancer activity. At the same time, the divergent sensitivity profiles of ROS-independent botanicals suggested that they have different mechanisms of action. This finding is of significant importance, with potential implications for cancer treatments using botanical compounds.

The anticancer activity of ROS-inducing botanicals was blocked by the presence of ROS scavengers in the cell cultures. This finding indicates that ROS induction is the primary mechanism responsible for the anticancer activity of these herbal products. Furthermore, all ROS-inducing botanicals showed equivocal anticancer activity in a relatively narrow concentration range, between 0.25 and $1 \mathrm{mg} / \mathrm{ml}$. This suggests that the anticancer potential (at least in vitro) of these botanicals is similar, and that the anticancer potential for ROS-inducing botanicals does not depend on the presence of specific components, but rather reflects the total content of the pro-oxidant phytochemicals.

The findings of research examining the ROS-sensitivity of different tumor types must be taken into consideration when designing a treatment plan that is botanical-based and tailored to the individual cancer subtype. The present findings demonstrated the potential for focusing on a select number of botanicals using sensitivity assays, which precludes the requirement for testing medicinal plants with similar mechanisms of action and potential sensitivities.

In summary, the present findings indicated that botanicals with anticancer activity are for the most part ROS-inducing, and that their cytotoxic effects reflect their total ROS content. As such, from an anticancer perspective, they may be interchangeable. On the other hand, the selection of botanicals for clinical use in traditional medicine takes into consideration a wide variety of factors, such as effects on the immune system and hematopoiesis, symptom relief, safety and bioavailability. There is a large body of research demonstrating these additional effects, with implications regarding the outcomes of conventional anticancer treatment $(2,3)$. These effects should play a role in the choice of botanicals to be used, as well as the findings of basic research on their anticancer activity. A better understanding of these mechanisms is required in order to develop evidence-based guidelines for cancer therapies. The current study results present a challenge to future research, which will need to examine the combined effects of ROS-dependent and -independent botanicals, as well as gaining a better understanding of whether this interaction is synergistic or inhibitory with respect to the interaction with conventional anticancer treatments. Finally, the classification of anticancer botanicals according to their ROS activity can serve as basis for a novel method of choosing botanicals for cancer treatment.

\section{Acknowledgements}

The authors would like to thank the Adelis Foundation for their support of the present research.

\section{References}

1. Cragg GM and Newman DJ: Plants as a source of anti-cancer agents. J Ethnopharmacol 100: 72-79, 2005.

2. Mok TS, Yeo W, Johnson PJ, Hui P, Ho WM, Lam KC, Xu M Chak K, Chan A, Wong H, et al: A double-blind placebo-controlled randomized study of Chinese herbal medicine as complementary therapy for reduction of chemotherapy-induced toxicity. Ann Oncol 18: 768-774, 2007. 
3. Zhang M, Liu X, Li J, He L and Tripathy D: Chinese medicinal herbs to treat the side-effects of chemotherapy in breast cancer patients. Cochrane Database Syst Rev CD004921, 2007.

4. Trachootham D, Alexandre J and Huang P: Targeting cancer cells by ROS-mediated mechanisms: A radical therapeutic approach? Nat Rev Drug Discov 8: 579-591, 2009.

5. Gibellini L, Pinti M, Nasi M, De Biasi S, Roat E, Bertoncelli L and Cossarizza A: Interfering with ROS metabolism in cancer cells: The potential role of quercetin. Cancers (Basel) 2: 1288-1311, 2010.

6. Cohen Z, Maimon Y, Yoeli-Lerner M, Yang P, Samuels N and Berger R: Selective anticancer effects and protection from chemotherapy by the botanical compoundLCS101: Implications for cancer treatment. Int J Oncol 46(1):308-316, 2015.

7. Fong S, Shoemaker M, Cadaoas J, Lo A, Liao W, Tagliaferri M, Cohen I and Shtivelman E: Molecular mechanisms underlying selective cytotoxic activity of BZL101, an extract of Scutellaria barbata, towards breast cancer cells. Cancer Biol Ther 7: 577-586, 2008.

8. Chen V, Staub RE, Baggett S, Chimmani R, Tagliaferri M, Cohen I and Shtiveldman E: Identification and analysis of the active phytochemicals from the anti-cancer botanical extract Bezielle. PLoS One 7: e30107, 2012.

9. Wang ZY, Wang DM, Loo TY, Cheng Y, Chen LL, Shen JG, Yang DP, Chow LW, Guan XY and Chen JP: Spatholobus suberectus inhibits cancer cell growth by inducing apoptosis and arresting cel cycle at G2/M checkpoint. J Ethnopharmacol 133: 751-758, 2011.
10. Tsai CF, Yeh WL, Huang SM, Tan TW and Lu DY: Wogonin induces reactive oxygen species production and cell apoptosis in human glioma cancer cells. Int J Mol Sci 13: 9877-9892, 2012.

11. Ou TT, Wu CH, Hsu JD, Chyau CC, Lee HJ and Wang CJ: Paeonia lactiflora Pall inhibits bladder cancer growth involving phosphorylation of Chk2 in vitro and in vivo. J Ethnopharmacol 135 162-172, 2011.

12. Hwang YJ, Lee EJ, Kim HR and Hwang KA: In vitro antioxidant and anticancer effects of solvent fractions from Prunella vulgaris var. lilacina. BMC Complement Altern Med 13: 310, 2013.

13. Liu Y, Xu Y, Ji W, Li X, Sun B, Gao Q and Su C: Anti-tumor activities of matrine and oxymatrine: Literature review. Tumor Biol 35: 5111-5119, 2014

14. Auyeung KK, Mok NL, Wong CM, Cho CH and Ko JK: Astragalus saponins modulate mTOR and ERK signaling to promote apoptosis through the extrinsic pathway in HT-29 colon cancer cells. Int J Mol Med 26: 341-349, 2010.

15. Sun JY, Yang H, Miao S, Li JP, Wang SW, Zhu MZ, Xie YH, Wang JB, Liu Z and Yang Q: Suppressive effects of swainsonine on C6 glioma cell in vitro and in vivo. Phytomedicine 16: 1070-1074, 2009.

16. Balsevich JJ, Ramirez-Erosa I, Hickie RA, Dunlop DM, Bishop GG and Deibert LK: Antiproliferative activity of Saponaria vaccaria constituents and related compounds. Fitoterapia 83: 170-181, 2012. 